\title{
LA PASTORAL DE LOS CUERPOS: POBREZA, GÉNERO Y ASISTENCIA HOSPITALARIA EN PUERTO RICO, 1812-1823
}

\author{
César Augusto Salcedo Chirinos \\ Universidad de Puerto Rico, Recinto de Río Piedras \\ Email: cesalchi@gmail.com \\ ORCID iD: https://orcid.org/0000-0003-3171-0307
}

Recibido: 20 abril 2018; Aceptado: 22 septiembre 2019

Cómo citar este artículo/Citation: Salcedo Chirinos, César Augusto (2020), "La pastoral de los cuerpos: Pobreza, género y asistencia hospitalaria en Puerto Rico, 1812-1823", Asclepio, 72(1): p302. https://doi.org/10.3989/asclepio.2020.11.

RESUMEN: En este artículo se analizan los primeros intentos realizados por el Estado para implementar la beneficencia pública en Puerto Rico. Se argumenta que los liberales lucharon por adelantar sus políticas en favor de la población desposeída, pero que sus acciones se vieron limitadas por las dificultades que encontraron en la Isla, entre ellas la falta de las instituciones de caridad. En los dos primeros periodos constitucionales (1812-1814 y 1820-1823) se intentó actuar en favor de todos los pobres, pero solo se logró atender a los enfermos pobres en los hospitales de la ciudad de San Juan. En el desarrollo del artículo se muestra que durante el siglo XIX importó mucho el género para ingresar a los hospitales: en el primer periodo constitucional solamente se asistieron a los hombres en el Hospital Militar; las mujeres fueron atendidas en el segundo periodo constitucional, cuando el Estado consiguió administrar el Hospital de Pobres. A pesar de la intermitencia entre los proyectos políticos liberales y conservadores, se dieron los primeros pasos para comenzar a asistir a los enfermos puertorriqueños pobres en los hospitales de la ciudad.

PALABRAS CLAVE: Liberalismo; beneficencia pública; pobreza; atención hospitalaria; género.

\section{THE PASTORAL OF THE BODIES: POVERTY, GENDER, AND HOSPITAL CARE IN PUERTO RICO, 1812-1823}

ABSTRACT: This article analyses the State's first attempt to implement public assistance in Puerto Rico. It is argued that liberals fought to advance their policies in order to favor the underprivileged population, but that their actions were limited by the difficulties encountered in the island, the lack of charities being one of them. During the first two constitutional periods (1812-1814 and 1820-1823,) an attempt was made to assist all of the underprivileged people, but only the sick at hospitals in the city of San Juan, were able to receive assistance. Throughout the article, it is demonstrated that during the XIX Century, gender played an important role when being admitted to a hospital: during the first constitutional period, only males received assistance at the Military Hospital; women received assistance during the second constitutional period, once the State was able to oversee the Hospital for the Underprivileged. In spite of the intermittence between the liberal and conservative political policies, the first steps to begin assisting sick and underprivileged Puerto Ricans in the city's hospitals were given.

KEY WORDS: Liberalism; public assistance; hospital care; poverty; gender. 


\section{INTRODUCCIÓN}

Eran las seis de la tarde del 14 de marzo de 1823 cuando el presbítero Francisco Correa se encontró con el abogado Aniceto Ruiz, quien también paseaba por el barrio Santa Bárbara, en la ciudad de San Juan de Puerto Rico. El hombre de Dios le contó al hombre de letras que en el campo posterior a la huerta del convento de los dominicos estaba abandonada una mujer enferma. Unas señoras le habían contado que llevaba todo el día tirada en medio del campo porque no tenía un lugar en donde recogerse. El doctor Ruiz se acercó a la mujer, una morena joven, y le preguntó las razones por las cuales se encontraba en aquellas condiciones. Ella le contestó que la habían echado a la calle porque le faltaban los cuatro reales para pagar la barraca que tenía alquilada; que había pedido socorro, pero nadie la había ayudado. Sin otra alternativa para evitar que aquella mujer permaneciera en la calle, el doctor Ruiz la llevó a su casa porque la madre de la joven tampoco podía recibirla; según explicaba la muchacha, el dueño del bohío en el que vivía su madre le había prohibido la entrada ${ }^{1}$.

Al día siguiente, el abogado contó aquella experiencia en la reunión mensual de la Junta de Beneficencia Municipal, de la cual era miembro. En ese momento argumentó, que, aunque la joven le causaba algunas incomodidades en su vida familiar, esperaba la pronta reapertura del Hospital de Pobres de la ciudad, que se encontraba en disputa con las autoridades eclesiásticas. María Paula, la joven enferma, recibió atención médica de manos del licenciado Emigdio Antique, quien también era miembro de la Junta de Beneficencia y luchaba por el restablecimiento del hospital. Dos semanas más tarde, María Paula murió en el seno de la familia que la había acogido, sin que aún se restableciera el Hospital de Pobres de la ciudad².

Una situación como la descrita, pudo ser común entre las mujeres pobres que enfermaban en la ciudad de San Juan de Puerto Rico a comienzos del siglo XIX: no recibían asistencia médica porque carecían de los medios necesarios para pagar por esos servicios. Esto sin contar el limitado número de facultativos que había en la ciudad. Por esa razón solían terminar en manos de los curanderos. Entre los límites de las murallas de la ciudad había dos hospitales, pero ninguno tenía autorización para recibir a las mujeres pobres enfermas. El Hospital Militar disponía de algunas camas para los hombres pobres del país y el Hospital de la Concepción, llamado comúnmente Hospital de
Pobres, recibía solo a hombres pobres. En el momento de la situación con María Paula, había dejado de recibir enfermos y era utilizado como residencia privada. Por estas razones se puede afirmar que las mujeres pobres que enfermaban en la ciudad, se encontraban con una dificultad para curarse y muchas terminaban pidiendo limosnas para sobrevivir. Es ahí donde radica la importancia de utilizar el concepto género para analizar la situación de la pobreza y la enfermedad; no era lo mismo ser hombre o mujer, al momento de entrar a un hospital.

Al analizar las actuaciones de los dos hombres que intervinieron en el caso de María Paula, se identifica que pareciera que se hubiesen desplazado los comportamientos esperados en cada uno de ellos. No fue el sacerdote quien recogió a la mujer necesitada y la llevó a su casa, sino el abogado; y cuando este lo hizo, no estaba pensando en el beneficio suyo, sino en el bien de la mujer enferma. Esta doble variación en el discurso de la caridad cristiana, que era el que imperaba en la Isla en aquel momento, se convierte en el punto de partida para analizar la incursión del discurso de la beneficencia pública en Puerto Rico. Estas ideas sobre la asistencia a los pobres como responsabilidad del Estado fueron parte del proyecto político liberal del siglo XIX, que llegó a Puerto Rico como consecuencia de la política constitucionalista. Esta distinción en la forma de concebir la ayuda que se daba a los pobres, a comienzos del siglo XIX, no aparece cuando Alfredo Montalvo Bardot habla de la historia de la caridad en la Isla; él se refiere indistintamente a la caridad privada y a la beneficencia pública, como si fueran lo mismo (Montalvo Bardot, 1995, pp. 150-1554). Para insistir en la diferencia de estos conceptos se afirma en el título de este artículo que el pastoreo, el cuidado de la población, ya no buscaba salvar las almas, sino los cuerpos; y que ese cuidado por el cuerpo ya no lo realizaba la Iglesia, sino que intentaba implementarlo el Estado.

Para desarrollar este planteamiento se toma como punto de partida el argumento foucaultiano que sostiene que gobernar, desde el siglo XVIII, era gobernar a la población. Este autor utilizó el concepto gubernamentalidad para referirse al proceso mediante el cual el Estado se dedicó a desarrollar las fuerzas productivas que le permitían subsistir (Foucault, 2006, pp. 135-136). Entre esas actividades estaba el control de las actividades que iban dirigidas a fomentar su fortalecimiento, entre las que se destacan la vigilancia sobre el número de habitantes, la satisfacción de sus necesidades vitales 
y el mantenimiento de su salud. Foucault llamó liberalismo a este nuevo juego de poder sobre la población, en donde se intentaba gobernar lo menos posible. No obstante, esa poca intervención, el Estado debió buscar un mecanismo de compensación para el caso de los pobres, por la amenaza que representaban para este proyecto económico. Ese mecanismo consistió en establecer una posibilidad para que los participantes del juego económico no perdieran todas las posibilidades de seguir jugando; es decir, se prevenían los efectos sociales extremos (Foucault, 2008, p. 208).

El propósito de este artículo es analizar los primeros intentos del Estado para organizar la beneficencia pública en Puerto Rico, los que se encontraron con ciertas limitaciones. Se sabe que el posterior desarrollo de esta beneficencia fue consecuencia de la implantación del régimen de las leyes especiales para las provincias, creado durante el reinado de Isabel II (1833-1868). El establecimiento de la Casa de Beneficencia de la Isla se realizó en 1844 (Rivera Rivera, 1995, p. 28; Rivera González, 2003, p. 61). Se evidencia que las acciones de la Iglesia local en favor de los pobres fueron mínimas, debido a la pobreza de esta institución, y que fueron las ideas modernas que llegaron con la política liberal las que intentaron implantar un sistema organizado de beneficencia, basado en la filantropía y no en la caridad. En la primera parte del artículo se exponen los conceptos que permiten explicar el nuevo modelo de asistencia que proponía el liberalismo como instrumento de control social. En la segunda parte se analiza el intento de implementar el sistema de beneficencia liberal durante el primer periodo constitucional (1812-1814), y se concluye que sus acciones se redujeron a la asistencia a los enfermos pobres en el Hospital Militar, porque era la única institución de la que podían disponer en ese momento. En la tercera parte se analiza el segundo intento durante el trienio constitucional (1820-1823), y se concluye que solamente se logró atender a los enfermos y a las enfermas pobres con la Hospitalidad Domiciliaria y con la Hospitalidad Pública, porque el Hospital de la Concepción era la única institución que estaba disponible para aplicar las nuevas políticas de asistencia. Con la reinstalación de este hospital, se abrió la posibilidad para que las mujeres pobres comenzaran a ser hospitalizadas.

\section{EL ESTADO, LA POBREZA Y EL CONTROL SOCIAL}

En los últimos años del siglo XVIII y en los primeros del siglo XIX, en Europa hubo unos cambios importantes en la forma de concebir al Estado. Con el paso del antiguo al nuevo régimen, el Estado asumió nuevas responsabilidades con relación a la población; especialmente con los que no podían satisfacer sus necesidades básicas para vivir. El pensamiento político liberal se preocupó por los pobres porque consideraba que esa parte de la población podía representar un problema para el nuevo orden social planificado, basado en el trabajo y en la producción. Por eso su énfasis en la promoción de las formas de organización que hicieran eficientes las ayudas que se le prestaban (Cardona, 2005, p. 32). Un hecho significativo en ese cambio de régimen fue el cuestionamiento de las funciones asistenciales que desempeñaba la Iglesia, lo que trajo como consecuencia el tránsito del discurso de la caridad cristiana al de la beneficencia pública, que fue asumida por el Estado como una responsabilidad suya (García Hourcade, 1996, p. 17).

Estos conceptos tenían unas distinciones precisas en el contexto general del siglo XIX. La caridad se concebía como una compasión exclusivamente cristiana, a partir de la cual se ayudaba al necesitado por el amor a Dios; mientras que la beneficencia se definía como una "compasión oficial que ampara(ba) al desvalido por un sentimiento de orden y justicia" (Arenal de García Carrasco, 1861, p. 43). Es decir, que la asistencia que se le prestaba a los pobres no tenía otra intención que la de mantener un orden social particular. Por eso, la filantropía fue una virtud respetada en la política liberal de este siglo, porque la asistencia que se daba al necesitado se consideraba que estaba inspirada en el amor a la misma humanidad, en la que se tomaba en cuenta la dignidad y el derecho del asistido y no el beneficio particular para el que ayudaba.

En la España de Carlos III (1759-1788) se pueden identificar algunas acciones dirigidas a implementar estas políticas: se recogía a los vagabundos y ociosos para llevarlos a los hospicios y obligarlos a trabajar. Ese nuevo orden social defendía ideas relacionadas con la riqueza, la paz y la prosperidad de la población; por eso se asistía a los pobres que pudieran trabajar, para que fueran útiles a la sociedad (Hernández Iglesias, 1876, p. 29). Para los pobres considerados inútiles para el trabajo, como los niños, los ancianos, los inválidos o los enfermos pobres, había otro tipo de ayuda, entre las que se contaban los hospitales y los hospicios. Es necesario aclarar que estos hospitales no formaban parte de una política sanitaria general para toda la población, sino dirigida únicamente a los pobres (Pons Pons y Vilar Rodríguez, 2014, p. 25). Una muestra de 
esos cambios que se fueron dando en España en la asistencia a los enfermos pobres se identifica en el reglamento que se estableció en Madrid en 1788, según el cual se creaba una normativa para asistir y curar a los pobres enfermos vergonzantes de esa ciudad (Maza Zorrilla, 1987, pp. 215-221).

Estos planteamientos políticos sobre la beneficencia pública comenzaron a generalizarse en el ámbito de los primeros periodos constitucionales. En la Constitución de Cádiz de 1812, en el contexto del primer periodo constitucional, se establecieron artículos relacionados con la asistencia a los necesitados. El 13 de junio de 1813 se aprobó La instrucción para el gobierno económico-político de las provincias, en la cual se encargaba a los Ayuntamientos de cuidar los hospitales, los hospicios y las casas de expósitos (Colección de decretos, 1820, p. 105). En el segundo periodo constitucional se articuló una legislación homogénea que garantizó el cuidado a los enfermos pobres. El 27 de diciembre de 1821 se firmó en un decreto el Reglamento General de Beneficencia Pública, en el cual se recomendaba la Hospitalidad Domiciliaria y la Hospitalidad Pública como formas de asistencia para los enfermos pobres (Gil Andrés, 2003, p. 94).

Los cambios relacionados con los asuntos de la beneficencia no se experimentaron en Puerto Rico hasta el primer cuarto del siglo XIX, cuando los avatares de la política liberal metropolitana repercutieron en la Isla. Este retraso podría estar relacionado, entre otras cosas, con la ausencia de las instituciones caritativas que en España administraba la Iglesia, porque la pobreza de la Iglesia local no le había permitido involucrarse en estos asuntos. La única institución caritativa que había administrado la autoridad eclesiástica isleña era el Hospital de la Concepción, que existía desde mediados del siglo XVI. Durante el siglo XVIII, el obispo Manuel Jiménez Pérez había intentado construir un nuevo hospital para los pobres de la ciudad, pero el proyecto se desvaneció cuando entregó el edificio construido para que se convirtiera en el Hospital Militar de la Isla (Arana Soto, 1976, p. 42). La única función específica identificada para asistir a los pobres de la ciudad la desempeñaba el Cabildo Eclesiástico a través del reparto de las limosnas que los fieles dejaban en sus testamentos. Estas limosnas se repartían en la puerta de la catedral sin ninguna sistematización y sin distinguir entre pobres enfermos y sanos, a pesar de que los testamentos eran muy claros en cuanto a quienes se deberían beneficiar.

Precisamente, a partir del análisis de estos testamentos se puede mostrar cómo el discurso de la ca- ridad privada, en la que se ayudaba a los pobres para ganar el cielo, continuaba existiendo en la Isla al momento de la creación de la Junta de Beneficencia en 1822 , cuando se promovió una ayuda a los pobres no relacionada con los premios celestiales. En estos testamentos se identifica que una parte importante de los bienes de los difuntos se dedicaban a pagar misas por el descanso de sus almas, y una pequeña parte, solo en algunos casos, la dejaban en beneficio de los pobres. La mayor parte de las herencias dejadas para estos pobres estaban dirigidas al mantenimiento del Hospital de Caridad de la ciudad. El padre Silvestre de Jesús Echavarría, en su testamento del año 1798 , dejó 600 pesos para que con los réditos se aumentaran 10 camas en ese hospital ${ }^{3}$; de igual manera, en 1818, el padre Pascual González dejó otros 200 pesos $^{4}$. Es importante indicar que el número de camas no aumentó a pesar de recibirse estas herencias. Pero no eran únicamente los eclesiásticos, los seglares también dejaban algo de sus bienes para esta institución. Doña María de la Soledad Franco, viuda de don Cristóbal Claro, enterrada el 5 de julio de 1818, dejó 200 pesos para socorro y alimentos de los enfermos del referido hospital ${ }^{5}$. Don Josep Dávila, regidor del Ayuntamiento de la ciudad, enterrado ese mismo día, dejó otros 10 pesos para esa función ${ }^{6}$.

En el contexto del segundo periodo constitucional se experimentó un cambio importante en la forma de compartir los bienes con los pobres, ya que no era necesario esperar el momento de la muerte para ayudar a los necesitados. Antes de reabrir el Hospital de Pobres, el 8 de septiembre de 1823, se recibieron donaciones que eran producto de las ganancias de obras de teatro realizadas con ese fin; después de la reapertura, se identifica otro tipo de donaciones. Aquí algunas de ellas: el padre Antonio Sánchez donó seis docenas de platos, una docena de vasos de vidrio, seis orinales y un jarrón; doña María del Carmen Andino entregó una porción de lienzos, una ponchera, dos escupideras de loza, un barril de vinagre y unas colchas nuevas, y don Luis Nebot, una caja de fideos, seis botellas de ron, seis botellas de vinagre y seis gallinas ${ }^{7}$.

Estos mismos testamentos son ocasión para distinguir los tipos de pobres que recibían las caridades con las cuales los difuntos esperaban recibir el cielo como premio. El padre José María Ruíz y Peña, enterrado el 29 de julio de 1818, especificó en su testamento que el valor de sus esclavos, sus bienes y sus muebles los dejaba para los pobres vergonzantes, y toda la ropa de su uso la dejaba para los pobres men- 
dicantes o pordioseros ${ }^{8}$. Así que distinguían entre pobres vergonzantes y mendicantes. El vergonzante era el pobre que procedía de un grupo social importante, que por alguna razón se había venido a menos y no tenía cómo satisfacer sus necesidades básicas; su origen le impedía salir a pedir limosnas de puerta en puerta, como hacían los otros pobres, porque el grupo social al que pertenecía les dictaba unas reglas de conducta (Guzmán Stein, 2009, pp. 230-231). El padre Ruiz y Peña reconocía que entre sus familiares había algunos venidos a menos, por eso solicitaba en su testamento que se les diera prioridad a ellos en el reparto de los bienes. El pobre mendicante era aquel que libremente pedía limosnas por la ciudad. Este pertenecía a grupos sociales inferiores y no tenía preocupación en que reconocieran su pobreza; es más, vivía de esa pobreza (Guzmán Stein, 2009, p. 231). Entre estos últimos pobres se consideraban a diferentes grupos, como viudas, huérfanos, ancianos, ciegos o inválidos. El testamento del padre don Lorenzo de Matos, máxima autoridad del Cabildo Eclesiástico de la Isla, sepultado el 3 de agosto de 1819, dejó en su testamento 100 pesos para que los distribuyeran de la siguiente manera: 25 para el hospital de pobres, 25 para los pobres vergonzantes, 25 para los pobres encarcelados y 25 para los pobres ancianos, ciegos e impedidos ${ }^{9}$.

Es importante aclarar que las acciones benéficas planificadas por el Estado para la isla de Puerto Rico, durante los dos primeros periodos constitucionales, fueron triplemente limitadas. Por un lado, la crisis económica que vivía la Isla en aquellos momentos afectó lo programado en la metrópoli para asistir a los pobres; por otro lado, fueron solamente los enfermos pobres los que recibieron la asistencia, no los pobres en general; y, por último, su breve duración en el tiempo. El Estado se valió de la única infraestructura que existía para implementar la legislación sobre la beneficencia, los hospitales de la ciudad. A continuación, se analiza cómo en el primer periodo constitucional se mejoraron algunas condiciones para los hombres pobres que enfermaban, y en el segundo periodo, para las mujeres pobres.

\section{LOS ENFERMOS POBRES EN EL PRIMER PERIODO CONSTITUCIONAL (1812-1814)}

El siglo XIX comenzó en Puerto Rico con una importante limitación para que los pobres pudieran curar sus enfermedades en algún hospital. El Hospital
Militar se negaba a recibir a los enfermos del país y el Hospital de la Concepción continuaba operando con muchas limitaciones, como lo había hecho la mayor parte del tiempo. El aumento de la población en la ciudad había reducido al mínimo la posibilidad para que los enfermos pobres pudieran ser admitidos en el llamado Hospital de Caridad. De hecho, entre 1783 y 1816 hubo un incremento significativo en la población de San Juan, que pasó de 6,462 habitantes a 8,907. (Martínez Vergne, 1999, p. 8). La población debía contar ordinariamente con suficientes bienes de fortuna para que los médicos pudieran atenderlos en sus propias casas. Miguel Xiorro, por ejemplo, dejó 300 pesos en su testamento para el doctor Tomás Prieto, médico mayor del Hospital Militar, por haberse dedicado a curar a su familia durante mucho tiempo $^{10}$. En las autorizaciones que el Ayuntamiento de la ciudad concedía para que se ejercieran la medicina y la cirugía en la Isla, se recomendaba la atención gratuita a los pobres; aún así, muchos pobres no contaban con los bienes necesarios para enfrentar el proceso de enfermarse.

Esta situación fue identificada en 1805 por el obispo Juan Alejo de Arizmendi y pretendió resolverla, como habían hecho sus predecesores, solicitando al Rey la unificación de los dos hospitales de la ciudad en el Hospital Militar, pero que cada cual conservara su propia administración. El argumento del obispo para elevar esa petición al Rey era que, como ese hospital había sido construido con fondos y limosnas del pueblo, los enfermos pobres debían beneficiarse de algo que les pertenecía. Esa había sido, precisamente, una de las exigencias del obispo Manuel Jiménez Pérez al momento de entregar el edificio en el año 1780 para que se atendieran las tropas del Rey: que los enfermos del país se beneficiaran de sus servicios. De Arizmendi repetía las exigencias del obispo Juan Bautista Zengotita: que se establecieran 50 camas para hombres de todas las clases, 12 camas para mujeres y 8 camas para enfermedades contagiosas. De esta manera se resolverían las limitaciones de espacio que existian en el Hospital de la Concepcion $^{11}$.

No obstante, la argumentación que presentó De Arizmendi, muchos pobres continuaron sin la posibilidad de ser atendidos en un hospital, especialmente las mujeres que no tenían cabida en ninguno de los dos que existían en la ciudad. La situación de la población pobre empeoró con la crisis económica que se agudizó como consecuencia de la suspensión del 
envío del Situado mexicano en 1810 (Sánchez Tarniella, 1984, p. 49). Por eso, entre las recomendaciones que las comunidades le dieron al diputado Ramón Power y Giralt para que presentara en las Cortes de Cádiz en 1810, estaba la falta de un hospital para atender a los pobres: "la numerosa población de la Isla, y la falta de arbitrios en los infelices, clama por un hospital más capaz y proporcionado que el que con el título de la Concepción existe en la ciudad" (Coll y Toste, 1914-1927, p. 103). En aquel ambiente adverso para que los enfermos pobres recibieran atención en los hospitales se proclamó la Constitución de Cádiz de 1812, en la cual se recomendaba a los Ayuntamientos que se encargaran de la policía de salubridad y de cuidar a los hospitales (Constitución política, 1812, art. 321).

El 20 de septiembre de 1812 se estableció el primer Ayuntamiento constitucional de la ciudad de San Juan, con el doctor Aniceto Ruiz y el teniente coronel del Ejército Juan Viñals como alcaldes de primer y segundo voto respectivamente. Entre las nuevas funciones que se le atribuyeron a este Ayuntamiento estaban las diputaciones de sanidad y policía médica, y la de obras pías, hospitales y establecimientos benéficos; eso que posteriormente se llamó salud y beneficencia. En estas nuevas funciones se identifica el carácter liberal del nuevo Ayuntamiento, en donde se asume, entre otras cosas, que la asistencia de la población era una responsabilidad del Estado (Actas del Cabildo, 1968, pp. 3-4). Con el concepto policía médica se referían al control de las enfermedades contagiosas, la organización y la supervisión del personal médico, la sanidad ambiental y la atención médica a los pobres que debía llevar ese Ayuntamiento (Rosen, 2005, p. 164).

Los diputados de sanidad y policía médica, Miguel Pizarro y Manuel de la Cruz, debían vigilar la salubridad de la ciudad; pero como carecían del conocimiento necesario para cumplir con esa función, solicitaron los servicios de un médico (Actas del Cabildo, 1968, p. 10). Varios días después se nombró a un graduado de la Universidad de Caracas, al licenciado Emigdio Antique, para que ejerciera la plaza de médico de la ciudad. Según ese nombramiento, debía colaborar con los diputados en la vigilancia de la mencionada salubridad. Ya el 20 de octubre de 1812 se veían los primeros resultados de esa vigilancia. El Ayuntamiento aprobó el aislamiento de María de la Concepción, una demente leprosa, para que pasara a vivir al Partido de Guaynabo. Se encargó a Eduardo Rodríguez para que la mantuviera aislada de la población, contando con una asignación de seis pesos mensuales. Los diputados expresaron que habían optado por esta medida porque la ciudad no contaba con un lugar especializado para los leprosos (Actas del Cabildo, 1968, p. 18).

El 4 de enero de 1813, el gobernador Salvador Meléndez Bruna firmó un decreto, según lo exigía la nueva Constitución política, para que se solicitara a las autoridades eclesiásticas que cedieran la administración del Hospital de la Concepción y lo pusieran a disposición de los enfermos pobres. Se solicitaban las "rentas, censos y fincas de que disfruta, y de cuanto pueda convenir a beneficio de tan piadoso establecimiento" (Actas del Cabildo, 1968, p. 51). En ese decreto se nombró al diputado José Romero para que negociara el traspaso con el obispo De Arizmendi. El argumento del Ayuntamiento para solicitar ese traspaso era la responsabilidad que tenía el Estado de conservar la salud de la población pobre. La autoridad eclesiástica se negó a entregar el hospital con el argumento de que como aquel establecimiento no funcionaba con fondos de la Real Hacienda, no podía ser cedido al Ayuntamiento ${ }^{12}$.

El poder que el Estado había desplegado en España para asumir las tareas que la Iglesia realizaba en beneficio de la población pobre, se encontró en Puerto Rico con una dificultad particular: la única institución relacionada con la Iglesia era el Hospital de Pobres. Por eso, cuando, por disposición de la regencia, el Ayuntamiento se reunió el 14 de enero de 1813 para analizar las obras que estaban en función de la felicidad y riqueza de la población, la discusión se centró en las obras que no existían. En ese momento, quedó demostrado que la Isla carecía de las instituciones básicas para prestarle ayuda a la población pobre. Entre las instituciones que se sugirieron que debían construirse estaban: un lazareto para aislar a los leprosos, que se financiara con los fondos del derecho de anclaje de los barcos; un cementerio que permitiera enterrar a los cadáveres en las afueras de las murallas; un hospicio que recogiera a los huérfanos desamparados, el cual podría funcionar en el edificio del Hospital de Caridad; una casa de misericordia que asilara a los mendigos y pordioseros, y una casa de recogidas que retuviera a las mujeres que se dedicaban a la prostitución. Con relación al Hospital de Caridad, se propuso que se mejorara el edificio existente, aunque continuaban los reclamos por el derecho de los pobres a ser admitidos en el Hospital Militar 
(Actas del Cabildo, 1968, pp. 55-60). Ninguno de estos proyectos, a excepción del cementerio, llegó a realizarse debido, entre otras cosas, a la crisis económica que vivía la Isla y la breve duración de la aplicación de la Constitución.

En el contexto de las limitaciones que identificó el Estado para asistir a los enfermos pobres hay que destacar que el mayor acierto se dio a partir del 12 de febrero de 1813, con la llegada del intendente Alejandro Ramírez Blanco, quien, procedente de Guatemala, venía a manejar la situación económica de la Isla. En un informe del 27 de octubre de 1815 comentaba, que una de las cosas que más lo conmovió a su llegada a Puerto Rico fue el clamor de los enfermos pobres porque carecían de un hospital en donde curar sus enfermedades y pedían que los admitieran en el Hospital Militar ${ }^{13}$. Al momento de asumir el cargo de Intendente de la Real Hacienda, el Hospital Militar estaba bajo el control del doctor Francisco Oller. Después de 1804, cuando murió el doctor Tomás Prieto, médico mayor del hospital, Oller, que era el cirujano mayor desde 1789 , permaneció como la única autoridad médica del hospital. Pudo ser este cirujano quien negó a los enfermos pobres del país la admisión a ese hospital. En el año 1809, el gobernador Meléndez Bruna insistía en que debía nombrarse al médico mayor del hospital, e indicaba que el estado general de la cirugía era lastimoso (Salcedo Chirinos, 2016, p. 65).

El intendente Ramírez sostenía en su informe que no pudo más que concederles un "socorro propio de la caridad cristiana y de la beneficencia paternal del Rey" a los enfermos pobres; es decir, permitió que los pobres fueran atendidos en el Hospital Militar, subsidiados por los fondos de la Real Hacienda. Comentaba que en 33 meses habían sido admitidos 109 enfermos, con un gasto de 1,638 pesos. Esta información fue enviada al Rey con el argumento de que en el Hospital de la Concepción no había cabida para todos estos enfermos ${ }^{14}$. El resultado final de las acciones de Ramírez a favor de los enfermos pobres fue el reconocimiento de 10 camas en el Hospital Militar, financiadas con los fondos de la Real Hacienda. Ese número de camas aumentó con el transcurso del tiempo. En 1839, ya eran 30 camas para los enfermos del país. El problema estaba en que en ese hospital no había cabida para las mujeres.

En el contexto de este primer periodo constitucional se nombró al doctor José Espaillat como médico mayor del Hospital Militar, el cual terminó por crear la primera Cátedra de Medicina que existió en Puerto Rico. El 30 de junio de 1814 se suspendió la aplicación de la Constitución de Cádiz y con ella algunos de los cambios que se habían iniciado a favor de los enfermos pobres. Lo que se salvó de todo lo iniciado en ese periodo fueron las 10 camas para los enfermos pobres en el Hospital Militar. El 11 de septiembre de 1815, el gobernador Meléndez Bruna visitó el Hospital de la Concepción, y en su informe destacaba que al llegar al lugar solamente había encontrado a un cuidador con tres enfermos, que el médico del hospital era el doctor Oller, pero que tenía más de un año sin visitarlo, y que en su lugar enviada a un practicante del Hospital Militar. Del edificio destacaba que el techo estaba en ruina ${ }^{15}$.

\section{LAS ENFERMAS POBRES EN EL SEGUNDO PERÍODO CONSTITUCIONAL (1820-1823)}

El 6 de marzo de 1820 se restableció en España la Constitución de 1812, y como consecuencia se reinstalaron los ayuntamientos constitucionales en Puerto Rico. El 16 de mayo siguiente, el gobernador Juan Vasco y Pascual juró lealtad a esa Constitución (Actas del Cabildo, 1978, p. 35). El 27 de diciembre de 1821, las Cortes extraordinarias firmaron la Ley de Beneficencia Pública, en la cual se afirmaba que las Juntas de Beneficencia se encargarían de cumplir las responsabilidades del Estado con relación a la asistencia de los pobres; por esa razón, ya los ayuntamientos no necesitaban crear comisiones especiales. Toda la normativa que regulaba estas juntas se recogía en el Reglamento General de Beneficencia Pública. Entre las funciones que este reglamento le atribuía a esas juntas estaban: informar a los ayuntamientos sobre los establecimientos de beneficencia; proponer y buscar fondos para la dotación de esos establecimientos, y ejecutar órdenes relacionadas con la mendicidad $^{16}$.

Ese reglamento distinguía cuatro tipos de establecimientos benéficos: Las Casas de Maternidad, Las Casas de Socorro, Los Hospitales de Enfermos, Convalecientes y Locos, y La Hospitalidad y Socorro Domiciliarios. Los dos primeros establecimientos estaban dirigidos a prestar ayuda a los que no podían satisfacer sus necesidades básicas para vivir, en los cuales tomaban en cuenta al ser humano desde su concepción hasta su deceso. Las Casas de Maternidad atenderían a las mujeres embarazadas y paridas, cuyos hijos hubiesen sido concebidos en una sexualidad 
llamada ilícita; en esas casas, los niños permanecerían hasta los seis años. En Las Casas de Socorro se atenderían a los pobres, desde los seis años hasta la vejez; se tomaban en cuenta especialmente a los huérfanos, los impedidos y los mendigos. Los últimos dos tipos de establecimientos estaban relacionados directamente con la curación de los enfermos pobres. La Hospitalidad Domiciliaria consistía en atender el proceso de la enfermedad en la casa del mismo enfermo, de manera que se limitara el número de los recluidos en los hospitales; mientras que la Hospitalidad Pública se dedicaría a los enfermos pobres que no tuvieran residencia o fueran portadores de enfermedades sospechosas. Aunque en la Hospitalidad Domiciliaria el médico debía visitar a los enfermos, el control de la asistencia estaba en manos de unos funcionarios que llamaban enfermeros. Este término, que no se utilizaba con la connotación contemporánea de curar al enfermo, se utilizaba para nombrar a quienes simplemente administraban aquel servicio. Toda la Hospitalidad Pública sí estaría bajo el control de los médicos en el hospital ${ }^{17}$.

El 8 de julio de 1822, el gobernador Francisco González de Linares envió el Reglamento General de Beneficencia Pública al Ayuntamiento de la ciudad para que le diera cumplimiento a la ley. El 22 de noviembre siguiente se constituyó la primera Junta de Beneficencia de San Juan. Los dos miembros del Ayuntamiento seleccionados como parte de ella fueron el doctor Francisco Pimentel, que por ser el alcalde de primer voto debía actuar como presidente, y el regidor Juan Severo Malagón. El clérigo nombrado por el Cabildo Eclesiástico fue Manuel Almanza. El médico elegido, quien ya había colaborado como médico de la ciudad en el primer periodo constitucional, fue el licenciado Emigdio Antique y el cirujano, el licenciado José Calvo. Los cuatro vecinos elegidos fueron: Aniceto Ruiz, quien también había participado en el proceso del primer periodo, Narciso Núñez, Francisco Matheu y Francisco Tadeo de Rivero ${ }^{18}$. Este último, comandante de Marina y miembro de la Sociedad Económica de Amigos del País, expresó en su carta de aceptación a la Junta que estaba identificado con el sistema constitucional, y que su deseo era cooperar con el bien y la prosperidad de esta Isla ${ }^{19}$. Estas ideas pueden relacionarse más con la filantropía que con la caridad cristiana.

El problema fundamental de la recién creada junta estaba relacionado con la falta de las instituciones que la Iglesia manejaba en la Península, así como la ausencia de los fondos necesarios para construirlas. Este problema es el punto de partida para explicar los pocos éxitos conseguidos por la beneficencia en Puerto Rico en el primer cuarto del siglo XIX. Entre los primeros fondos obtenidos por la Junta se cuenta el traspaso del Hospicio de las 15 Marías, el cual había sido fundado por el padre José María Ruiz y Peña, y que en esos momentos estaba custodiado por su hermano Aniceto Ruiz. Cuando este custodio colocó los bienes de ese hospicio a disposición de la Junta de Beneficencia, expuso claramente el motivo por el cual lo hacía: “...manifestando que todos los establecimientos destinados a objetos de beneficencia pública no mencionados en la ley, deberían suprimirse según el artículo 134, adjudicándose sus fondos a los que queden existiendo"20. Entre los demás fondos se cuentan las limosnas recogidas en la ciudad, las mandas forzosas de los testamentos y lo obtenido en los sorteos de la lotería y las funciones de teatro, además de las donaciones particulares de importantes personalidades de la ciudad.

Los primeros esfuerzos de la Junta de Beneficencia se dirigieron a la instalación de una Casa de Socorro, que recogiera a todos los mendigos de la ciudad; la cual se pensaba establecer en algunos de los salones del convento de los franciscanos, recién expropiado y convertido en escuela. Esta casa no llegó a instalarse. La ley ordenaba que mientras se prepararan esas Casas de Socorro, había que controlar la presencia de los mendigos en las calles. El reglamento establecía que después de verificar quiénes fueran verdaderamente pobres, se le expidieran unas licencias para mendigar. Estos autorizados para pedir, eran los que llamaban pobres de solemnidad. En la realización de los exámenes médicos a estos pobres, fue que las autoridades descubrieron que había algunos tan enfermos que necesitaban algo más que un permiso para mendigar. El primer caso fue el de Marcelina Álvarez. Ella se presentó a la Junta a finales de enero de 1823 para pedir un permiso para mendigar: era una mujer mayor con sus achaques; su marido, también anciano, y su único hijo estaban inmóviles en sus lechos. El licenciado Antique confirmó que el anciano padecía una parálisis general y el hijo, un efecto reumático crónico que era incurable. El médico recomendó que aquella familia recibiera una ayuda en su casa porque no había quien aportara lo básico para vivir $^{21}$.

En la sesión del 8 de marzo de 1823, la Junta acordó encargarse de asistir a los enfermos pobres en sus 
casas $^{22}$. Al considerar esta fecha para argumentar a favor de la existencia de la Hospitalidad Domiciliaria en Puerto Rico, a principios del siglo XIX, se niega la fecha del 18 de junio de 1822 que propone Lidio Cruz Monclova (Cruz Monclova, 1979, p. 208). En la programación de esta asistencia a los enfermos pobres, se aprovechó la división de la ciudad en cuatro barrios, que existía desde finales del siglo XVIII. Para la asistencia del barrio Santo Domingo se nombró al doctor José Espaillat, para San Francisco al doctor José María Vargas, para Santa Bárbara al licenciado Emigdio Antique, y para San Juan al doctor Francisco Oller. Como enfermeros fueron nombrados dos vocales de la Junta de Beneficencia, Antonio Guerrero y Aniceto Ruiz; cada uno de ellos recibiría 25 pesos para asistir a los enfermos en sus casas. Al final de cada semana, debían informar sobre los gastos y el número de enfermos curados o muertos ${ }^{23}$. Es importante aclarar que estos enfermeros no eran soldados, como sostiene Lydia Pérez González (Pérez González, 1997, p. 37). Ya se ha comentado que Aniceto Ruiz era abogado.

Esta atención a los enfermos pobres en sus casas, la llamada Hospitalidad Domiciliaria, representó un doble beneficio para el Ayuntamiento de la ciudad, porque se les prestaba ayuda a unos enfermos pobres sin tener que estar en un hospital, y se evitaba, al mismo tiempo, que estuvieran como mendigos en las calles. Entre los atendidos podían incluirse las mujeres. En los últimos cuatro meses de esta asistencia (23 de agosto al 15 de diciembre de 1823) se atendieron 56 pobres, con un gasto de 94 pesos aproximadamente. Dos de aquellos enfermos murieron mientras recibían la ayuda ${ }^{24}$.

Según el acta de la sesión del 5 de abril de 1823, en la realización de los exámenes para otorgar las licencias a los mendigos, se presentó Florentina Campos, una leprosa pobre con su hijo de 12 años, quien también era leproso. Conforme al Reglamento de Beneficencia, este caso no era para autorizarle a mendigar, sino para llevarlo al hospital. El problema estaba en que en el Hospital Militar no se recibían mujeres y el Hospital de la Concepción se había convertido en una residencia familiar. En estas circunstancias, la Junta de Beneficencia pidió al Ayuntamiento que apresurara a las autoridades eclesiásticas para que entregaran el Hospital de Pobres, porque de acuerdo con la recién aprobada ley, era el Estado el que debía administrar ese hospital. En esa misma fecha, el licenciado Antique informaba que había examinado a otras cinco mujeres enfermas y consideraba que podían restablecerse con la atención médica en el hospital, porque de lo contrario morirían. En este contexto fue cuando el doctor Aniceto Ruiz llevó a María Paula a su casa porque no había un hospital que la admitiera. La esperanza de la Junta estaba puesta en obtener la administración del Hospital de Pobres para atender aquellas mujeres enfermas. Desde el 14 de diciembre de 1822, la Junta de Beneficencia había solicitado al Ayuntamiento que tramitara la entrega del referido hospital, pero el edificio se encontraba ocupado por la familia del coronel de Artillería don Rafael Riesch, un emigrado de Venezuela, quien había movido todas sus influencias para impedir la entrega ${ }^{25}$.

El 12 de julio de 1823, las autoridades eclesiásticas entregaron el Hospital de la Concepción con todos los bienes que poseía. Al recibirlo, la Junta comenzó las reparaciones necesarias para poner a disposición de aquellas mujeres enfermas las ocho camas con que contaba ese hospital. Una de las primeras acciones en función de la apertura, fue la elaboración de un reglamento para su funcionamiento ${ }^{26}$. Este instrumento de gobierno fue concebido desde los planteamientos de la medicina moderna, según los cuales el hospital dejaba de ser un lugar para morir, y se convertía en un lugar para curar, en donde el médico tendría el control de todas las actividades (Foucault, 1996, pp. 107-116). Según este reglamento, el hospital tendría un director, un capellán, un administrador, un médico o cirujano, un practicante, un cabo de sala y algunas enfermeras. El administrador se encargaría, además, de llevar los libros, de suministrar ropas, muebles, utensilios, ornamentos, así como también de cobrar las rentas. El médico o cirujano visitaría diariamente y trataría la condición de cada enferma, determinaría la salida de las curadas y supervisaría la ventilación, limpieza y fumigación del edificio. El practicante acompañaría al médico en sus visitas, anotaría sus indicaciones y repartiría medicamentos y haría las curaciones. El cabo de sala mantendría el orden entre las enfermas, y las enfermeras o asistentes se encargarían de limpiar y atender las necesidades de las enfermas ${ }^{27}$.

Un hecho significativo en el proceso de traspaso del hospital fue la cantidad de donaciones recibidas; una de las más destacadas por la Junta fue el ofrecimiento de las medicinas para el hospital que hizo el licenciado Francisco Sellés, un farmacéutico emigrado de Venezuela. La acción fue calificada con un acto 
filantrópico. Es necesario aclarar que las donaciones no solo fueron en moneda, sino que también se recibieron utensilios y alimentos. Entre ellas puede destacarse una cantidad importante de ron, el cual se utilizaba en las curaciones ${ }^{28}$.

El Hospital de la Concepción comenzó a operar nuevamente el 8 de septiembre de $1823^{29}$. El director fue Antonio Guerrero, quien había ejercido como enfermero en la Hospitalidad Domiciliaria; el capellán y administrador, el presbítero Lorenzo de Sotomayor, quien se había desempeñado como capellán de este mismo hospital; el médico fue el doctor Francisco Oller, quien había sido nombrado por el obispo Francisco de la Cuerda en 1794; el practicante fue Joaquín Avendaño, el cabo de sala Antonio Blanco, y las enfermeras Basilia y Marcela Elías y Candelaria y Dionisia Lara. Mientras comenzaron a ingresar las enfermas al Hospital de Pobres, continuó ofreciéndose la Hospitalidad Domiciliaria. En el informe del 6 de septiembre de 1823, los enfermeros decían que en la última semana habían gastado 24 pesos en atender 11 enfermos y que uno de ellos había muerto.

Las acciones de la Junta de Beneficencia, dirigidas especialmente a implementar la legislación en favor de los pobres, se concentraron en la atención a los enfermos pobres, a esa parte de la población que carecía de los medios para enfrentar los procesos de las enfermedades. Por esa razón se enfocaron en la Hospitalidad Domiciliaria y en la Hospitalidad Pública, porque disponían, entre otras cosas, de la arquitectura necesaria para alcanzar ese fin. El Hospital de la Concepción representó la posibilidad para tomar en cuenta a las mujeres pobres enfermas.

En aquel contexto en el que florecía la atención hospitalaria a los enfermos pobres de la ciudad, el 4 de diciembre de 1823 llegó una noticia del Ayuntamiento: la Junta de Beneficencia debía cesar en sus funciones porque el Rey se había restablecido en la plenitud de su soberanía. La última sesión se realizó el 13 de diciembre de ese año. En ella se acordó entregar el Hospital de la Concepción a las autoridades eclesiásticas nuevamente; especialmente el dinero que se había obtenido de la venta de la casa del
Hospicio de las 15 Marías $^{30}$. No obstante, el regreso del Hospital de Pobres a las autoridades eclesiásticas, su dedicación al cuidado de las mujeres enfermas pobres continuó durante el siglo XIX.

\section{CONCLUSIÓN}

Los cambios en la forma de comprender la asistencia a los pobres, en el tránsito del antiguo al nuevo régimen, trajeron como consecuencia que el Estado tuviera una nueva actitud ante esa parte de la población. Ayudar al pobre era salvar a la sociedad y al nuevo proyecto político, porque el liberalismo no podía excluir a esa parte de la población. En Puerto Rico, hubo que esperar hasta mediados del siglo XIX para que comenzaran a cristalizarse aquellas ideas sobre la beneficencia pública: fue en 1844 cuando se inauguró la Casa de Beneficencia. Aquellos primeros intentos en favor de la beneficencia encontraron algunas limitaciones, entre otras cosas, la falta de instituciones de caridad para desarrollar el proyecto y la brevedad con que se ejerció el poder constitucionalista. A pesar de esas limitaciones, estos primeros intentos abrieron un campo de acción para que los enfermos pobres de la ciudad de San Juan encontraran cabida en los hospitales de la ciudad. En el primer periodo constitucional los hombres pobres consiguieron ser atendidos en el Hospital Militar, y en el segundo periodo fueron las mujeres pobres. Esas conquistas en los espacios hospitalarios se mantuvieron durante el siglo XIX. El Hospital Militar admitió una buena cantidad de enfermos locales, los que eran necesariamente pobres, y el Hospital de la Concepción cambió el nombre y recibió a las mujeres pobres enfermas. A partir del segundo período constitucional, comenzó a considerarse un hospital de mujeres. En la descripción de la ciudad de San Juan que hizo Pedro Tomás de Córdova en 1845, lo presentaba como el "Hospitalillo con la advocación de la Concepcion para las mujeres" (Coll y Toste, 1914-1927, pp. 21-22). El 5 de enero de 1887 comenzó a ser administrado por las Siervas de María, una congregación religiosa dedicada a la atención a los enfermos (Hernández Aponte, 2013. p. 38). 


\section{NOTAS}

1 Actas de la Junta de Beneficencia, Archivo General de Puerto Rico (en adelante AGPR), Fondo Documentos Municipales, Serie San Juan, Caja 82, Fol. 38

2 Actas de la Junta de Beneficencia, AGPR, Fondo Documentos Municipales, Serie San Juan, Caja 82, Fol. 39v.

3 Testamento de Silvestre de Jesús Echavarría, Archivo General de Indias (en adelante AGI), Santo Domingo, Leg. 2524, Fol. 597. (Consultado en el Centro de Investigaciones Históricas, Universidad de Puerto Rico, Recinto de Río Piedras, Carrete 140).

4 Testamento de Pascual González, Archivo Histórico Arquidiocesano (en adelante AHA), Parroquia Catedral, Fondo Nuestra Señora de los Remedios, Sección Sacramental, Serie Defunciones, Libro 23 (1818-1820), Apartado 27, Fols. $9 \mathrm{v}-11$.

5 Testamento de Pascual González, AHA, Parroquia Catedral, Fondo Nuestra Señora de los Remedios, Sección Sacramental, Serie Defunciones, Libro 23 (1818-1820), Apartado 27, Fols. 9v-11.

6 Testamento de Josef Dávila, AHA, Parroquia Catedral, Fondo Nuestra Señora de los Remedios, Sección Sacramental, Serie Defunciones, Libro 23 (1818-1820), Apartado 27, Fols. 8-8v.

7 Actas de la Junta de Beneficencia, AGPR, Fondo Documentos Municipales, Serie San Juan, Caja 82, Fols. 100-106.

8 Testamento de José María Ruiz y Peña, AHA, Parroquia Catedral, Fondo Nuestra Señora de los Remedios, Sección Sacramental, Serie Defunciones, Libro 23 (1818-1820), Apartado 27, Fols. 9-9v.

9 Testamento de Lorenzo de Matos, AHA, Parroquia Catedral, Fondo Nuestra Señora de los Remedios, Sección sacramental, Serie Defunciones, Libro 23 (1818-1820), Apartado 27 , Fols. 83-85.

10 Testamento de Miguel Xiorro, AGI, Santo Domingo, Leg. 2524, Fol. 604v. (Consultado en el Centro de Investigaciones Históricas, Universidad de Puerto Rico, Recinto de Río Piedras, Carrete 140)

11 Expediente informativo instruido para esclarecer el derecho del ramo de guerra a la propiedad del edificio destinado al Hospital Militar, AGPR, Fondo Gobernadores Españoles, Serie Hospital-Indulto, Caja 238, Fols. 120-123v.

12 Expediente sobre la administración y dirección del Hospital de la Concepción de Puerto Rico y desavenencias entre el gobernador y el obispo, AGI, Santo Domingo, Legajo 2520, Fols. 24-27. (Consultado en el Centro de Investigaciones Históricas, Universidad de Puerto Rico, Recinto de Río Piedras, Carrete 75)

13 Expediente informativo instruido para esclarecer el derecho del ramo de guerra a la propiedad del edificio destinado al Hospital Militar, AGPR, Fondo Gobernadores Españoles, Serie Hospital-Indulto, Caja 238, Fols. 251-253.
14 Expediente informativo instruido para esclarecer el derecho del ramo de guerra a la propiedad del edificio destinado al Hospital Militar, AGPR, Fondo Gobernadores Españoles, Serie Hospital-Indulto, Caja 238, Fols. 251-253.

15 Visita del Hospital de la Concepción por el gobernador Salvador Meléndez Bruna, AGI, Ultramar, Leg. 431, Fols. 528-530. (Consultado Centro de Investigaciones Históricas, Universidad de Puerto Rico, Recinto de Río Piedras, Carrete 209).

16 Expediente sobre el nombramiento de la Junta de Beneficencia, AGPR, Fondo Documentos Municipales de San Juan, Serie Beneficencia, Leg. 26, Exp. 2.

17 Expediente sobre el nombramiento de la Junta de Beneficencia, AGPR, Fondo Documentos Municipales de San Juan, Serie Beneficencia, Leg. 26, Exp. 2.

18 Actas de la Junta de Beneficencia, AGPR, Fondo Documentos Municipales, Serie San Juan, Caja 82, Fol. 1.

19 Expediente sobre el nombramiento de la Junta de Beneficencia, AGPR, Fondo Documentos Municipales de San Juan, Serie Beneficencia, Leg. 26, Exp. 2.

20 Actas de la Junta de Beneficencia, AGPR, Fondo Documentos Municipales, Serie San Juan, Caja 82, Fol. 2v.

21 Actas de la Junta de Beneficencia, AGPR, Fondo Documentos Municipales, Serie San Juan, Caja 82, Fols. 21v-22v.

22 Actas de la Junta de Beneficencia, AGPR, Fondo Documentos Municipales, Serie San Juan, Caja 82, Fol. 36v.

23 Actas de la Junta de Beneficencia, AGPR, Fondo Documentos Municipales, Serie San Juan, Caja 82, Fol. 37.

24 Actas de la Junta de Beneficencia, AGPR, Fondo Documentos Municipales, Serie San Juan, Caja 82, Fols. 102v, 104v, 105v, 106, 111v y 114.

25 Expediente que contiene la copia impresa del expediente formado por la Junta Municipal de Beneficencia en reclamo del Hospital de la Concepción, AGPR, Fondo Documentos Municipales de San Juan, Leg. 26, Exp. 4.

26 Actas de la Junta de Beneficencia, AGPR, Fondo Documentos Municipales, Serie San Juan, Caja 82, Fols. 85-90.

27 Expediente sobre el nombramiento de la Junta de Beneficencia, AGPR, Fondo Documentos Municipales de San Juan, Serie Beneficencia, Leg. 26, Exp. 2.

28 Actas de la Junta de Beneficencia, AGPR, Fondo Documentos Municipales, Serie San Juan, Caja 82, Fol. 101v.

29 Expediente que contiene el acta y oficios del Cabildo Eclesiástico sobre entrega a la Junta Municipal de Beneficencia del Hospital de Caridad, AGPR, Fondo Documentos Municipales de San Juan, Serie Beneficencia, Leg. 26. Exp. 5.

30 Expediente sobre la entrega del Hospital de Caridad, archivos y demás pertenencias de la Junta de Beneficencia, por cesación de esta, AGPR, Fondo Documentos Municipales de San Juan, Serie Beneficencia, Leg. 26, Exp. 6. 


\section{BIBLIOGRAFÍA}

Actas del Cabildo de San Juan Bautista de Puerto Rico, 18121814 (1968), Puerto Rico, Publicación Oficial del Municipio de San Juan.

Actas del Cabildo de San Juan Bautista de Puerto Rico, 18201821 (1978), Puerto Rico, Publicación Oficial del Municipio de San Juan.

Arana Soto, Salvador (1976), El hospital de Puerto Rico (Historia del Hospital Militar), San Juan, Asociación Médica de Puerto Rico.

Arenal de García Carrasco, Concepción (1861), La beneficencia, la filantropía y la caridad, Madrid, Imprenta del Colegio de Sordo-Mudos y Ciegos.

Cardona, Álvaro (2005), La salud pública en España durante el trienio liberal, 1820-1823, Madrid, Consejo Superior de Investigaciones Científicas.

Colección de los decretos y órdenes que han expedido las Cortes Generales y Extraordinarias, desde el 24 de febrero de 1813 hasta septiembre de este mismo año. (1820), Tomo IV, Madrid, Imprenta Nacional.

Coll y Toste, Cayetano (1914-1927), Boletín histórico de Puerto Rico, Tomos IX y X. San Juan, Tipografía Cantero, Fernández \& Co.

Constitución política de la monarquía española (1812), Cádiz, Imprenta Real.

Cruz Monclova, Lidio (1979), Historia de Puerto Rico (XIX) 18081868, Tomo I. Río Piedras, Editorial Universitaria.

García Hourcade, José Jesús (1996), Beneficencia y sanidad en el siglo XVIII: el Hospital de San Juan de Dios de Murcia, Murcia, Universidad de Murcia.

Gil Andrés, Carlos (2003), "Sistema de beneficencia", en: Jaime Alvar Ezquerra (Coord.), Diccionario de historia de España, Madrid, Ediciones Istmo, pp. 94-95.

Guzmán-Stein, Miguel (2009), "Benefactores, pobres mendicantes y pobres vergonzantes: Filantropía y caridad en las relaciones sociales de Costa Rica", en: Ronny J.Viales Hurtado (ed.), Pobreza e historia en Costa Rica. Determinantes estructurales y representaciones sociales del siglo XVIIII a 1950, San José, Editorial UCR, pp. 207-272.

Hernández Aponte, Gerardo Alberto (2013), La salud y la labor social: ministerio de las Siervas de María Ministras de los Enfermos en Puerto Rico (1887-1921), San Juan, Instituto de Cultura Puertorriqueña.
Hernández Iglesias, Fermín (1876), La beneficencia en España, Tomo I, Madrid, Establecimiento tipográfico de Manuel Minuesa.

Foucault, Michel (1996), "Incorporación del hospital a la tecnología moderna", en: La vida de los hombres infames, La Plata, Editorial Altamira, pp. 107-120.

Foucault, Michel (2006), Seguridad, territorio, población, México, Fondo de Cultura Económica.

Foucault, Michel (2008), Nacimiento de la biopolítica, México, Fondo de Cultura Económica.

Maza Zorrilla, Elena (1987), Pobreza y asistencia social en España, siglos XVI-XIX, Valladolid, Universidad de Valladolid.

Martínez-Vergne, Teresita (1999), Shaping the Discourse on Space Charity and Its Words in Nineteenth Century San Juan, Puerto Rico, Austin, University of Texas Press.

Montalvo Bardot, Alfredo (1995), "El Estado y la ética de la caridad en Puerto Rico: 1898-1903", Revista de Ciencias Sociales, Vol. 164, Núm. 3-4, pp. 149-164.

Pérez González, Lydia (1997), Enfermería en Puerto Rico, desde los precolombinos hasta el siglo XX, Mayagüez, Universidad de Puerto Rico.

Pons Pons, Jerònia; Vilar Rodríguez, Margarita (2014), El seguro de salud privado y público en España. Su análisis en perspectiva histórica, Zaragoza, Prensa de la Universidad de Zaragoza.

Rivera González, María de Lourdes (2003), “La Casa de Beneficencia: espacio de caridad y castigo para la infancia puertorriqueña (1844-1920). Historia y Sociedad, 14, pp. 53-81.

Rivera Rivera, Antonia (1995), El Estado español y la beneficencia en el Puerto Rico del siglo XIX, República Dominicana, Editorial El Cuervo Dorado.

Rosen, George (2005), De la policía médica a la medicina social. Ensayos sobre la historia de la atención a la salud, México, Siglo XXI Editores.

Salcedo Chirinos, César Augusto (2016), Las negociaciones del arte de curar. Los orígenes de la regulación de las prácticas sanitarias en Puerto Rico (1816-1846), Lajas, Editorial Akelarre.

Sánchez Tarniella, Andrés (1984), Obras Completas, Tomo II, San Juan, Ogún de los Hierros. 